Case Report

\title{
Coronary Artery and Pulmonary Artery Fistula Originated from Significant Stenosis in the Left Anterior Descending Artery
}

\begin{abstract}
Alper Sami Kunt
Cardiovascular Surgery Clinic, Yasam Hastanesi, 1486 Sokak, Muratpasa, Antalya, Turkey

Correspondence should be addressed to Alper Sami Kunt; dralper@msn.com

Received 21 December 2012; Accepted 13 January 2013

Academic Editors: W. D. Grant, F. Natale, and M. Sand

Copyright (C) 2013 Alper Sami Kunt. This is an open access article distributed under the Creative Commons Attribution License, which permits unrestricted use, distribution, and reproduction in any medium, provided the original work is properly cited.

Coronary artery fistula (CAF) is defined as a rare anomalous connection between a coronary artery and a major vessel or a cardiac chamber. We report a case of a left anterior descending coronary (LAD) stenosis and coronary artery fistula between the LAD coronary artery and the pulmonary artery (PA). CAF is often diagnosed by coronary angiogram. We describe our diagnostic approach and review the literature on the epidemiology, the pathophysiology, the diagnostic modalities, and the treatment options.
\end{abstract}

\section{Background}

Coronary artery fistulas between the LAD and the PA are rare congenital malformations; however, concomitant significant coronary artery stenosis can cause coronary steal phenomenon and this results in myocardial ischemia [1].

\section{Case Presentation}

A 64-year-old female was admitted at our hospital for management of unstable angina. Coronary angiography revealed severe atherosclerotic coronary artery disease associated with an LAD-PA fistula (Figure 1). Coronary artery catheterization showed proximal stenosis in the left anterior descending coronary artery. Physical examination revealed continuous murmur. Ejection fraction was measured as $65 \%$. After providing informed consent, the patient underwent coronary artery bypass grafting through a sternotomy in May 2011, without cardiopulmonary bypass. Left internal thoracic artery was anastomosed to the left anterior descending coronary artery. After identification of the LAD-PA fistula, it was sutured on both sides with 4/0 polypropylene (Figure 2 ). Special attention was directed to the absence of thrill, which may suggest the possibility of residual fistula, and the thrill was disappeared manually. The patient had an uneventful postoperative course and was discharged on postoperative day 6 in good clinical condition. During 11 months of followup, the patient had an excellent quality of life without subsequent cardiac events.

\section{Conclusions}

Fistula between coronary artery and pulmonary artery is a rare congenital anomaly. It was first described by Krause in 1865 , but the first successful surgical treatment was described by Fell and colleagues [2] in 1958.

Coronary artery fistula causes myocardial ischemia both by producing a coronary steal and by imposing an additional volume load on the left ventricle. However, these patients with coronary artery fistula are entirely asymptomatic $[1,3-$ 5]. If the fistula is associated with atherosclerotic coronary artery disease, angina pectoris may be one of the symptoms and although it is rare, myocardial infarction may also develop. Our patient had both severe coronary artery disease and coronary fistula those aggravates each other's clinic.

There is no specific medical therapy for coronary fistula, yet in some cases the fistula can be treated by transcatheter embolization or surgical intervention [6-8]. In our patient, the LAD-PA fistula was an incidental diagnosis associated with the significant coronary artery disease, and both conditions were surgically treated. The main goal was to perform coronary artery bypass grafting and then close the fistula through both sides with ligation during the same procedure 


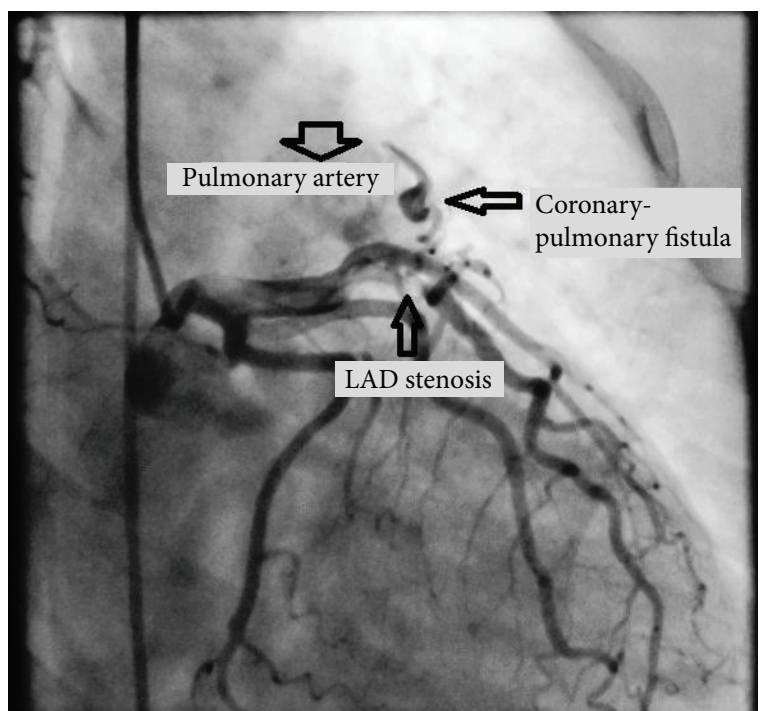

FIGURE 1: Angiographic view of coronary fistula between left internal thoracic artery and pulmonary artery.

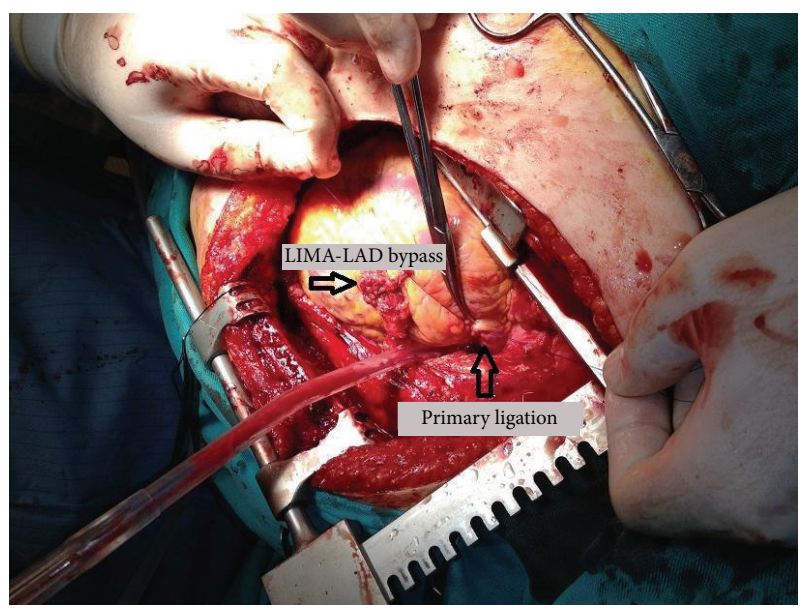

Figure 2: Coronary bypass and fistula ligation.

while heart beating. The operation was completed by the favorable postoperative course.

In conclusion, surgery with or without cardiopulmonary bypass is still the best method of treatment for the association between coronary fistula and severe coronary artery disease.

\section{Abbreviations}

LAD: Left anterior descending

PA: Pulmonary artery.

\section{Consent}

Written informed consent was obtained from the patient for publication of this case report and any accompanying images. A copy of the written consent is available for review by the Editor-in-Chief of this journal.

\section{Conflict of Interests}

A. S. Kunt declares that he has no conflict interests.

\section{Authors' Contribution}

A. S. Kunt is the author and the surgeon. A. S. Kunt read and approved the final paper.

\section{References}

[1] M. Vavuranakis, C. A. Bush, and H. Boudoulas, "Coronary artery fistulas in adults: incidence, angiographic characteristics, natural history," Catheterization and Cardiovascular Diagnosis, vol. 35, no. 2, pp. 116-120, 1995.

[2] E. H. Fell, J. Weinberg, A. S. Gordon, B. M. Gasul, and F. R. Johnson, "Surgery for congenital coronary artery arteriovenous fistulae," A.M.A. Archives of Surgery, vol. 77, no. 3, pp. 331-335, 1958.

[3] J. E. Lowe and D. C. Sabiston Jr., "Congential malformations of the coronary circulation," in Gibbon's Surgery of the chest, D. C. Sabiston Jr. and F. C. Spencer, Eds., pp. 1409-1424, WB Saunders, Philadelphia, Pa, USA, 4th edition, 1983.

[4] T. M. Daniel, T. P. Graham, and D. C. Sabiston Jr., "Coronary artery-right ventricular fistula with congestive heart failure: surgical correction in the neonatal period," Surgery, vol. 67, no. 6, pp. 985-994, 1970.

[5] E. A. Rittenhouse, D. B. Doty, and J. L. Ehrenhaft, "Congenital coronary artery cardiac chamber fistula: review of operative management," Annals of Thoracic Surgery, vol. 20, no. 4, pp. 468-485, 1975.

[6] J. Meyer, G. J. Reul, and C. E. Mullins, "Congenital fistulae of the coronary arteries: clinical considerations and surgical management in 23 patients," Journal of Cardiovascular Surgery, vol. 16, no. 5, pp. 506-511, 1975.

[7] J. A. Ogden and H. C. Stansel Jr., "Coronary arterial fistulas terminating in the coronary venous system," Journal of Thoracic and Cardiovascular Surgery, vol. 63, no. 2, pp. 172-182, 1972.

[8] D. B. Effler, W. C. Sheldon, J. J. Turner, and L. K. Groves, "Coronary arteriovenous fistulas: diagnosis and surgical management. Report of fifteen cases," Surgery, vol. 61, no. 1, pp. 41$50,1967$. 


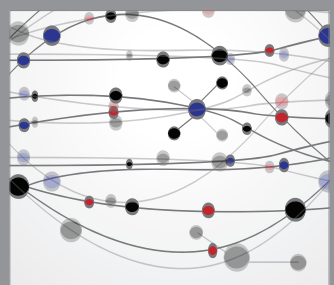

The Scientific World Journal
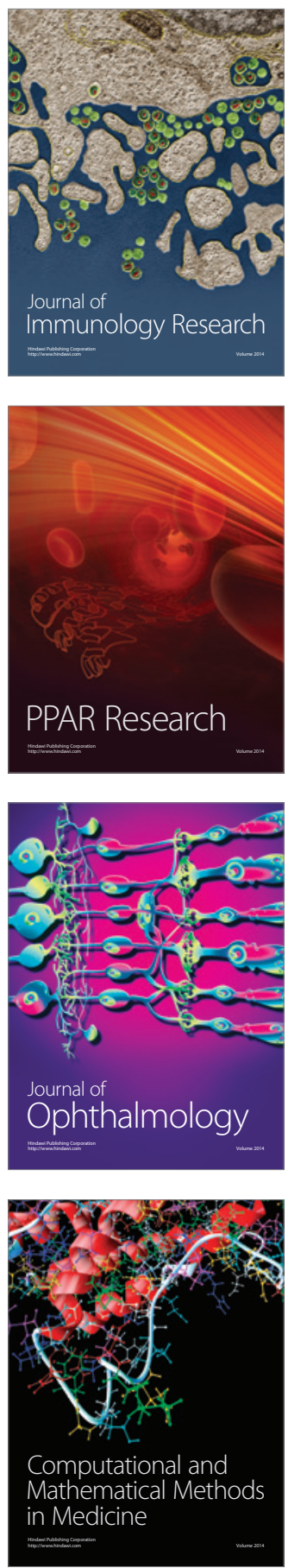

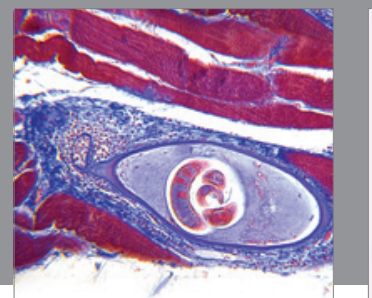

Gastroenterology

Research and Practice
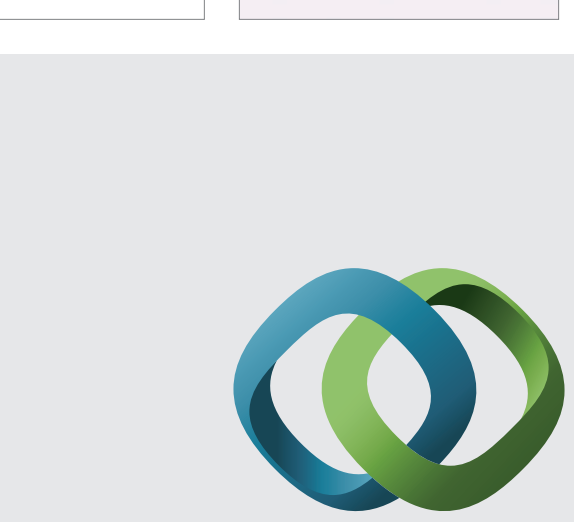

\section{Hindawi}

Submit your manuscripts at

http://www.hindawi.com
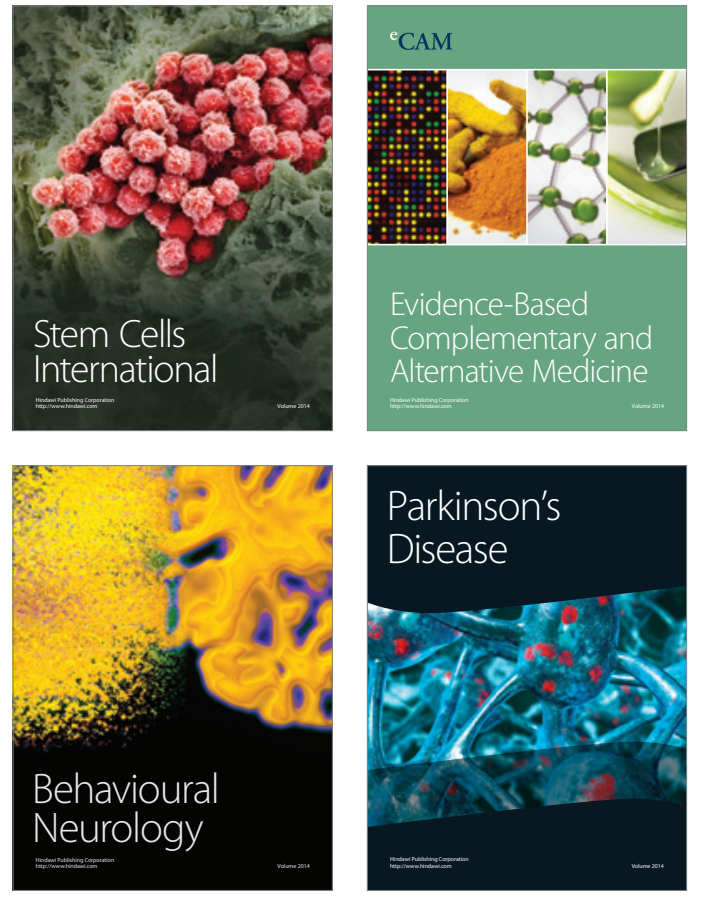
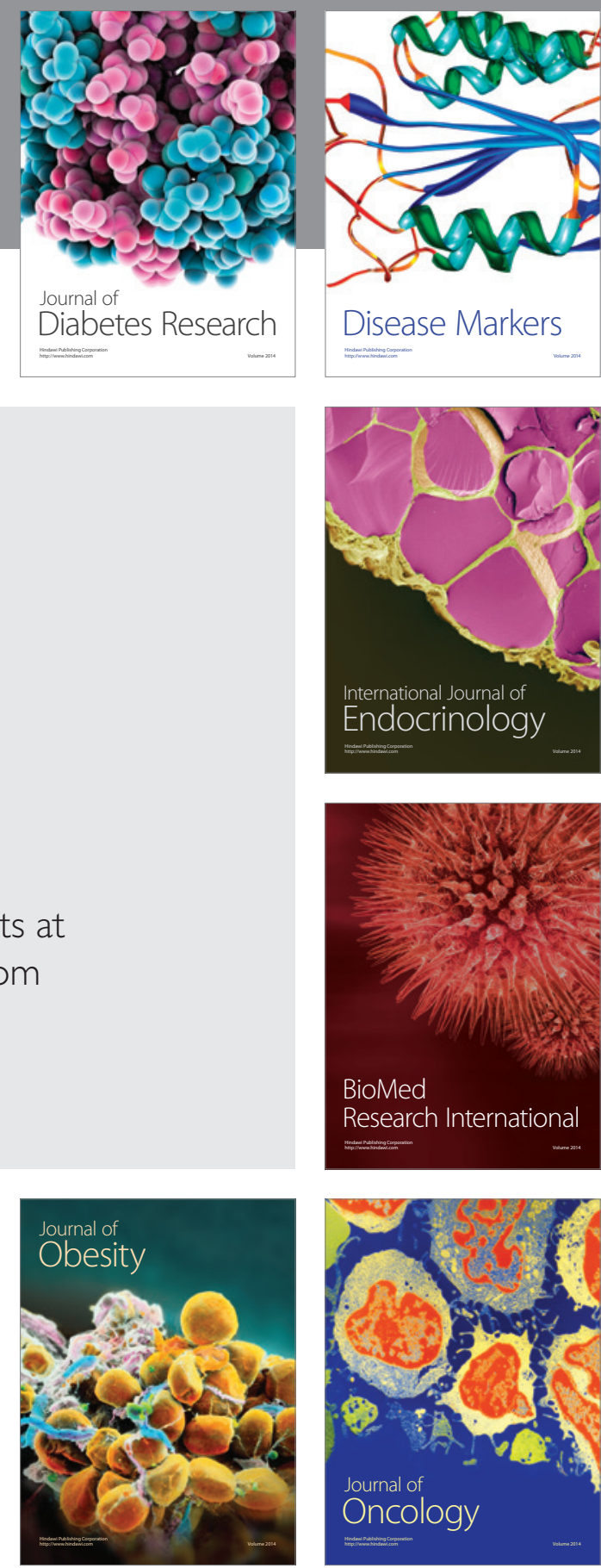

Disease Markers
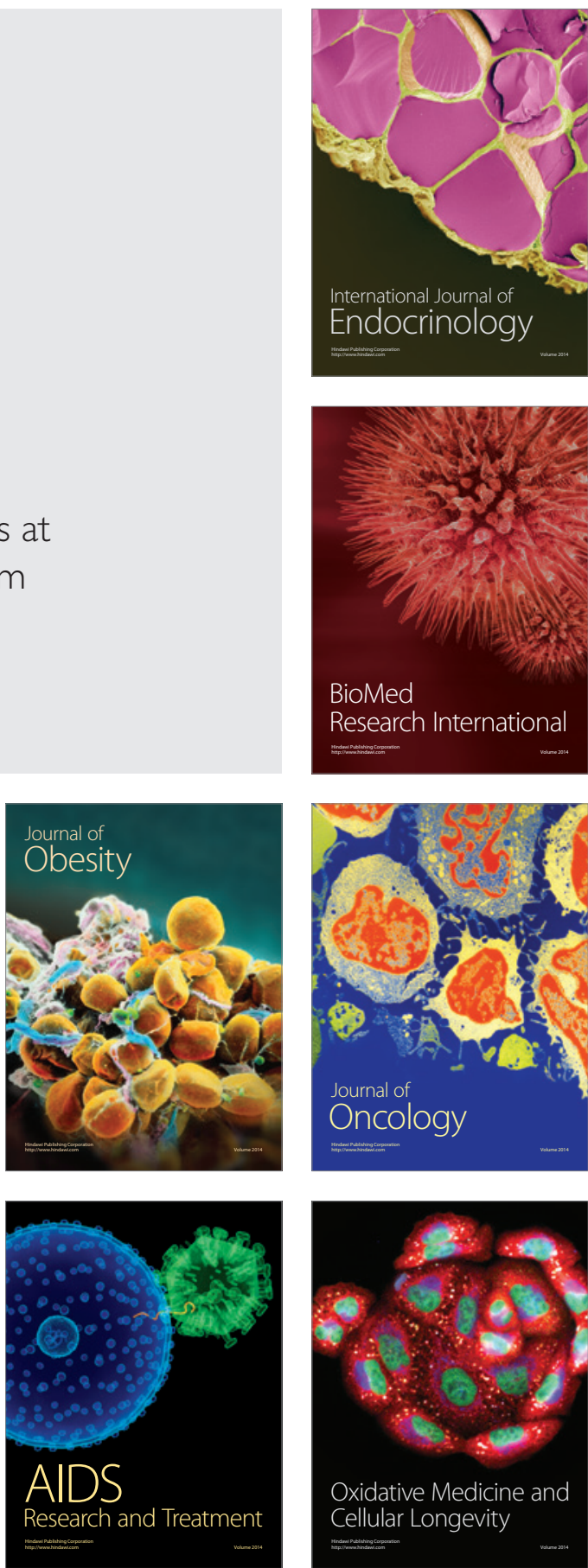